\title{
Survival in progressive supranuclear palsy and frontotemporal dementia
}

Wang Zheng Chiu ${ }^{1}$, Laura Donker Kaat ${ }^{1}$, Harro Seelaar ${ }^{1}$, Sonia M. Rosso ${ }^{1}$, Agnita J.W. Boon ${ }^{1}$,

Wouter Kamphorst ${ }^{2}$, John C. van Swieten ${ }^{1}$

${ }^{1}$ Department of Neurology, Erasmus Medical Centre, Rotterdam, the Netherlands

${ }^{2}$ Department of Neuropathology, Vrije Universiteit Medical Centre, Amsterdam, the Netherlands

Correspondence to: Dr. John C. van Swieten

Erasmus University Medical Centre Rotterdam

Department of Neurology, Room Hs 611

‘s-Gravendijkwal 230

3015 CE Rotterdam, the Netherlands

Tel: 00311070438 22, Fax: 0031107044727

E-mail: j.c.vanswieten@erasmusmc.nl

Word count (excluding title page, abstract, references, figures and tables): 3391

Number of references: 41

Number of tables, figures: 2, 2

Words that cover main aspects of this article:

Progressive supranuclear palsy, frontotemporal dementia, frontotemporal lobar degeneration, survival, PSP Rating Scale 


\section{Abstract}

Objective: To compare survival and to identify prognostic predictors for progressive supranuclear palsy and frontotemporal dementia.

Background: PSP and FTD are related disorders. Homozygosity for H1 haplotype is associated with PSP, whereas several MAPT mutations have been identified in FTLD-tau. Survival duration probably reflects underlying pathophysiology or disease.

Methods: Patients with PSP and F'TD were recruited by nation-wide referral. Survival of 354 FTD patients was compared to that of 197 PSP patients. Cox regression analysis was performed to identify prognostic predictors. F'TLD-tau was defined as Pick's disease and FTDP-17 with MAPT mutations. Semiquantitative evaluation of tau-positive pathology was performed on all pathologically proven cases.

Results: Median survival of PSP patients (8.0 years; 95\% CI 7.3 to 8.7 ) was significantly shorter than of FTD patients (9.9 years; 95\% CI 9.2 to 10.6). Corrected for demographic differences, PSP patients were still significantly more at risk of dying than F'TD patients. In PSP, male gender, older onset-age, and higher PSP Rating Scale score were identified as independent predictors for shorter survival, whereas in FTD a positive family history and an older onset-age were associated with a poor prognosis. The difference in hazard rate was even more pronounced when comparing pathologically proven cases of PSP with F'TLD-tau.

Conclusion: Survival of PSP patients is shorter than of FTD patients, and probably reflects a more aggressive disease process in PSP. Independent predictors of shorter survival in PSP were male gender, older onset-age and higher PSP rating scale score, whereas in F'TD a positive family history and higher onset-age were predictors for worse prognosis. 


\section{Introduction}

Progressive supranuclear palsy (PSP) is clinically characterized by parkinsonism, supranuclear gaze palsy and cognitive decline, $[1,2]$ and shows clinical, pathological and genetic overlap with frontotemporal dementia (FTD).[3-5] A frontal presentation has been identified in 20 percent of PSP cases, [6] whereas F'TDP-17 associated with microtubule associated protein tau (MAPT) mutations may present with the clinical picture of PSP.[7, 8] Neuronal and glial tau-positive inclusions are found in PSP and consist mainly of hyperphosphorylated four-repeat tau isoforms. In contrast to this, a subset of FTLD with Pick bodies (so-called Pick's disease) is characterized by the accumulation of three-repeat tau isoforms, [9] whereas inclusions in FTDP-17 with $M A P T$ mutations variably consist of three- and four-repeat tau isoforms, depending on the location of the mutation. [10] The relevance of clinical and pathological overlap is further emphasized by the strong association between MAPT H1/H1 genotype and PSP.[11] Determining survival within this F'TLD-PSP spectrum is of important clinical relevance and may give insight into the underlying disease process. However, only a few small studies compared survival between PSP and F'TD and did not find any differences. $[12,13]$ Small pathological series of PSP and taupositive and tau-negative FTLD patients have shown conflicting results regarding the effect of tau pathology on survival.[14-16] In a recent study, specific neuropsychological profiles in FTLD have been correlated to disease duration, whereas onset-age or positive family history were not.[12] Early falls and gaze palsy have been found as prognostic features in retrospective studies on PSP, [17-20] whereas the PSP Rating Scale (PSPRS) has also proven to be of predictive value in survival in a prospective longitudinal study.[21] However, this still has to be replicated. Severity of tau pathology has shown an inverse correlation with prognosis in PSP, [22, 23] whereas conflicting results have been reported in F'TLD.[14-16, 24]

The aim of this study is to prospectively investigate the survival in two large cohorts of PSP and FTD patients in relationship to demographic and clinical features, and to the presence and severity of tau pathology in a subset of patients who underwent brain autopsy. 


\section{Methods}

Patients with PSP and F'TD were recruited by nation-wide referral from neurologists and by visiting patients in nursing homes. $[6,25]$ Detailed clinical history, including the first presentation of symptoms, was obtained from patients and their family members, and by reviewing medical records. The onset-age was defined as the age at which the first symptom attributable to PSP and FTD appeared according to the patient's caregiver and from medical records. In case of discrepancies, data from medical records were used. Data on family history were obtained using a structured questionnaire provided by spouse or first-degree relative. Family history was defined positive if at least one first-degree relative suffered from dementia, parkinsonism or motor neuron disease. All available hard copies of neuroimaging of both PSP and FTD patients were reviewed by the investigators in order to exclude other structural causes of both conditions and to semiquantitatively measure the severity of lobar atrophy.

PSP patients were neurologically examined, videotaped and the severity of their cognitive and motor functioning was scored by means of Mini-mental State Examination (MMSE), Frontal Assessment Battery (FAB), Unified Parkinson's Rating Scale-III (UPDRS-III) and PSPRS. FTD patients underwent neurological examination, neuropsychological evaluation and neuroimaging (CT, MRI or SPECT with 99m'Tc-hexamethyl propyleneamine oxime (HMPAO)). The clinical diagnosis of all patients was established in a consensus meeting according to the National Institute for Neurological Diseases and Stroke-Society for PSP (NINDS-SPSP) criteria[2] and the Lund and Manchester criteria for F'TD.[26] PSP patients were subdivided according to phenotype as described by Williams et al.[27] 119 patients were classified as $\underline{\text { Richardson's syndrome (RS) and seven cases of PSP-parkinsonism (PSP-P) were identified in our }}$ cohort. Of 18 patients there was insufficient data available of the first two years after onset. The remainder of the patients ( $n=51$ ) could not be subdivided into a phenotype. Both studies on PSP and FTD patients were approved by the Medical Ethics Committee of the Erasmus Medical Centre of Rotterdam. Informed consent for participation (including blood collection) was 
obtained from the spouse or a first-degree relative of each patient. MAPT, CHMP2B and GRN genes were sequenced in all familial FTD patients, as has been previously described.[28-30] In PSP patients with a positive family history, screening of $M A P T, G R N$, and $L R R K 2$ was performed according to previously described methods.[29, 31, 32]

The possibility of post-mortem examination was discussed with patients and their relatives during follow-up. Brain autopsy of patients who gave consent and who died during follow up was conducted by the Netherlands Brainbank according to their Legal and Ethical Code of Conduct. All brains that became available for autopsy were processed for routine staining and immunohistochemistry with AT8 (1:40, Innogenetics, Ghent, Belgium), ubiquitin (1:500, Dako, Glostrup, Denmark), three-repeat tau isoform (RD3) and four-repeat tau isoform (RD4), p62 (BD Biosciences Pharmingen, San Diego, CA, USA; $1: 200$, following $80^{\circ} \mathrm{C}$ antigen retrieval), TDP-43 (Proteintech, Chicago, IL, USA; 1 : 100, following pressure-cooking), $\beta$-amyloid (anti- $\beta$ amyloid, DAKO, Glostrup, Denmark, 1: 100, following formic acid pre-treatment), $\alpha$-synuclein (anti- $\alpha$-synuclein, Zymed Laboratories, San Francisco, California, USA; undiluted, following formic acid pretreatment). These were incubated overnight at $4{ }^{\circ} \mathrm{C}$. Endogenous peroxidase activity was inhibited by 30 min incubation in PBS-hydrogen peroxide-sodium azide solution (100 $\mathrm{ml} 0.1 \mathrm{M}$ PBS, $2 \mathrm{ml} \mathrm{30 \%} \mathrm{H2O2,1} \mathrm{ml}$ natriumazide). The Histostain-Plus broad-spectrum kit DAB (Zymed, San Francisco, CA, USA) was used as a detection system. Slides were counterstained with Mayer's haematoxylin and mounted in Entellan. The neuropathological diagnosis FTLD was classified into FTLD-tau and FTLD-U (with or without TDP-43-positive inclusions).[33] F'TLD-tau was defined as Pick's disease and FTDP-17 with MAPT mutations. Cases with FTD-MND were excluded from this study. In FTLD-tau cases, neuronal loss and tau-staining reactive neurons and glial cells were visually quantified (none, mild, moderate and severe) in the following regions: frontal lobe, temporal lobe, hippocampus, parietal lobe, caudate nucleus and substantia nigra. 
The neuropathological diagnosis PSP was established according to international criteria,[34] and a semiquantitative assessment of neurofibrillary tangles (NFT), tufted astrocytes (TA), oligodendroglial coiled bodies (CB) and thread pathology (Th) in all regions was carried out by two raters (WK, JvS) using a five-point grading scale according to Williams et al.[23] The PSP-tau score was calculated from the combined grade of coiled bodies and thread lesions in the substantia nigra and caudate and dentate nucleus.

Follow-up of PSP and FTD patients was performed by visits to the outpatient department of the Erasmus Medical Centre or by telephone interview with relatives up to August 1, 2008.

\section{Statistical analysis}

SPSS 15.0 for Windows (SPSS, Chicago, IL) was used for analysis. Onset-age, gender and family history were analyzed by independent sample $t$-test or Chi-square test. Actuarially corrected median survival was calculated, as well as mean survival in deceased cases. Survival analysis was performed using the Cox proportional hazard model, using a backward selection procedure model. Only results of multivariate analyses are shown, with variables that were significant in the univariate analysis. As the early occurrences of clinical symptoms are incorporated into the PSPRS, these symptoms were not analyzed together with the PSPRS in one model. However, different sections of the PSPRS (history, mentation, bulbar, ocular, limb and gait sections) were analyzed separately. Entry date was set as time of first symptoms. Censoring date was either date of death or end of follow-up (August 1st, 2008). The assumption of proportionality of hazards was examined by Log-Log plots. Hazard ratios (HR) and 95\% confidence intervals (CI) were calculated. Onset-age and PSPRS score were categorized into quartiles. Correlation between tau pathology and disease duration and onset-age was examined using Spearman's calculation. All statistical testing took place at a 0.05 level of significance (two-tailed). 


\section{Results}

The demographic data of patients with PSP and F'TD are summarized in Table 1.

\begin{tabular}{llll}
\hline \multicolumn{4}{l}{ Table 1 Demographic characteristics of patients with PSP and FTD } \\
\hline & PSP & FTD & p-value \\
N & 197 & 354 & \\
Age at symptom onset, years* & $66.2 \pm 8.1$ & $57.5 \pm 8.9$ & $<0.001$ \\
Male gender, n (\%) & $102(51.8)$ & $164(46.3)$ & 0.220 \\
Presence of family history, n (\%) & $\mathbf{6 2 ( 3 1 . 5 )}$ & $169(47.7)$ & $<0.001$ \\
\hline *Mean \pm SD. PSP, progressive supranuclear palsy; FTD, frontotemporal dementia. & \\
\hline
\end{tabular}

F'TD-MND patients $(n=30)$ were excluded, due to their known shorter disease duration. Two PSP patients died of non-natural cause and have not been included in the survival analyses. The mean onset-age and age at death of PSP patients were significantly higher than that of F'TD patients. During follow-up, 133 of 197 patients with PSP died at a mean disease duration of $7.2 \pm$ 2.6 years, whereas 242 out of 354 FTD patients had died after a mean disease duration of $9.2 \pm$ 4.1 years.

Sumival and hazard analysis of PSP and FTD

The median disease duration in PSP patients (8.0 years; 95\% CI 7.3 to 8.7 ) was significantly shorter than in FTD patients (9.9 years; 95\% CI 9.2 to 10.6) (Chi-square 17.1, p<0.001) (Figure 1). This worse prognosis for PSP patients than F'TD patients in a univariate analysis (HR 0.634; 95\% CI 0.509 to 0.788 ) remained significant after adjustment for gender, onset-age and family history (HR 0.766; 95\% CI 0.603 to 0.975). Comparing PSP phenotypes, RS (6.8 years; $95 \%$ CI 6.3 to 7.4 ) was found to have shorter median survival than PSP-P (10.9 years; $95 \%$ CI 7.5 to 14.2 ) and the non-conclusive group (8.8 years; $95 \%$ CI 8.2 to 9.3 ). Cox proportional hazards regression model of PSP patients (Table 2) revealed male gender, older onset-age ( $>72$ years) and higher score on the PSPRS to be independent predictors for 
shorter disease duration, after adjustment for the interval between onset and ascertainment (mean of $5.3 \pm 2.6$ years).

Table 2 Multivariate Cox models in PSP patients (HR with 95\% CI)

\section{p-value}

Gender

$$
0.627(0.428 \text { to } 0.918) \quad 0.016
$$

Positive family history

ns

\section{Estimated onset-age}

$<62$

62 to 66

66 to 72

$>72$

\section{PSPRS*}

0 to 35

35 to 48

48 to 62

$>62$
1 (reference)

$0.878(0.515$ to 1.498$) \quad 0.634$

1.453 (0.846 to 2.498$) \quad 0.176$

$2.028(1.211$ to 3.398$) \quad 0.007$

*10 patients had missing values on the PSPRS;

HR, Hazard ratio; CI, confidence interval; ns, not significant

When entering separate sections of the PSPRS in the model, only supranuclear ocular motor exam (IR 1.195; 95\% CI 1.090 to 1.310) remained significant, whereas bulbar exam (HR 1.144; 95\% CI 0.997 to 1.312 ) and gait exam (HR 1.063; $95 \%$ CI 0.999 to 1.131 ) were near significant.

In FTD patients, positive family history (HR 1.438; 95\% CI 1.114 to 1.858 ) and onset-age $>64$ years (HR 1.656; 95\% CI 1.160 to 2.363) were significantly associated with poor survival. Looking into family history in F'TD in more detail, mean disease duration of deceased FTD patients with a negative family history (9.9 years; $95 \%$ CI 9.1 to 10.6 ) was significantly longer than that of FTD patients with a positive family history (8.4 years; 95\% CI 7.7 to 9.1; $\mathrm{p}=0.006$ ). In this latter group a trend towards longer mean disease duration of patients with a $M A P T$ mutation $(\mathrm{n}=36$ from 10 families; 9.3 years; $95 \%$ CI 7.8 to 10.8 ) was found compared to patients without a $M A P T$ mutation ( $\mathrm{n}=83$, including 17 patients with a GRN mutation from three families; 8.1 
years; 95\% CI 7.3 to $8.8 ; \mathrm{p}=0.105)$. Of the MAPT mutations, L315R had the shortest mean disease duration ( $n=5 ; 5.7 \pm 1.9$ years), followed by P301L ( $n=20 ; 8.2 \pm 3.0$ years), whereas $\mathrm{R} 406 \mathrm{~W}$ had the longest mean disease duration ( $\mathrm{n}=4 ; 17.5 \pm 3.2$ years). The remaining $M A P T$ mutations, S320F ( $\mathrm{n}=1), \mathrm{G} 272 \mathrm{~V}(\mathrm{n}=5)$, and $\Delta \mathrm{K} 280(\mathrm{n}=1)$ all had a mean disease duration of just above 10 years. Patients with a GRN mutation had a survival of $7.7 \pm 2.8$ years.

\section{Pathology}

Pathological examination was available for 24 PSP patients (all RS) and 61 F'TLD patients (FTLD-tau n=32 and FTLD-U n=29). Men were over-represented $(70.8 \%)$ in the PSP series, and the FTLD series showed a higher percentage of a positive family history $(57.6 \%)$ and younger onset-age (55.3 years) than the total group, due to significant lower onset-age for cases with MAPT mutations (50.9 years).

After adjustment for gender, onset-age, and family history, FTLD-tau patients remained less at risk than PSP patients (IHR 0.524; 95\% CI 0.282 to 0.974), and a trend towards longer survival was found compared to FTLD-U patients (HR 0.608; 95\% CI 0.361 to 1.024).

The F'TLD-tau group consisted of 15 sporadic cases, all of which showed pure three-repeat tau pathology, and 17 cases with $M A P T$ mutation, with pure three-repeat (G272V and $\triangle \mathrm{K} 280$ ), pure four-repeat (P301L), or a mix of three-repeat and four-repeat (S320F, R406W and L315R) tau pathology depending on the location of the mutation. All PSP cases showed four-repeat tau pathology. The mean disease duration of sporadic Pick's disease cases was 12.1 years and was similar to that in $M A P T$ cases with three-repeat tau pathology $(n=6)$ of 10.2 years, whereas a trend $(\mathrm{p}=0.098)$ could be observed towards shorter survival in MAPT cases with 4-repeat tau pathology $(\mathrm{n}=7$ ) of 8.6 years. Disease duration in MAPT mutations with a mix of three-repeat and four-repeat tau pathology varied considerably.

The FTLD-U cohort consisted of nine type 1, 16 type 2 and four type 3 (all four with GRN mutation) cases. Survival of pathological GRN cases did not significantly differ from the total 
group of deceased GRN cases or FTLD-U type 2 cases ( $8.4 \pm 3.1$ years), but was significantly

shorter than survival of F'TLD-U type 1 cases $(11.6 \pm 5.0$ years $)$.

\section{Tau pathology quantification}

Neuronal loss in PSP cases was most prominent in subthalamic nucleus, globus pallidus, dentate nucleus and substantia nigra. Tau pathology consisting of globoid neurofibrillary tangles, tufted astrocytes and glial coiled bodies varied considerably between cases, with the subthalamic nucleus, thalamus, substantia nigra, basal pontine nuclei, locus coeruleus and dentate nucleus regions most severely involved. The severity of tau pathology, expressed in the tau-score, showed a significant negative correlation with disease duration (Figure 2), but was not correlated with onset-age.

For the FTLD-tau group, neuronal loss in frontal, temporal and hippocampus regions was severe in most cases, whereas parietal, caudate nucleus and substantia nigra regions showed a variable neuron loss. Tau-positive inclusions showed a similar pattern of topographic distribution with severe tau pathology in frontal and temporal cortex and hippocampal regions, whereas the severity of tau pathology was more variable in parietal cortex, caudate nucleus and substantia nigra. Astrocytic tau pathology was severe in the L315R mutation, but only mild in other $M A P T$ mutations and sporadic Pick's disease. No significant correlation could be found between disease duration and either neuronal loss or tau-reactivity in any region.

\section{Discussion}

This study is the largest prospective population-based study comparing the survival between patients with PSP and FTD, and showed significantly shorter disease duration in PSP. This difference was even more pronounced when comparing pathologically proven cases of PSP with FTLD-tau. This study replicates, for the first time, the prognostic value of the PSPRS with a sharp increase of probability of death above a score $>60$. In PSP patients, male gender and older 
onset-age were also independent predictors for shorter disease duration, whereas a positive family history and an older onset-age were associated with a poor prognosis for F'TD.

Our observation of a shorter disease duration in PSP than in FTD contrasts with two other studies, $[12,13]$ in which the small number of PSP patients may explain the lack of correlation. Our findings are probably close to true survival rates, as the patients were population-based ascertained. Looking into the natural history of PSP separately, the mean disease duration of deceased cases of 7.2 years in the present study comes very close to 6.8 years found in the only other large prospective study by Golbe et al.,[21] whereas a large retrospective study[20] showed shorter survival of 5.7 years. This was also true for RS cases in the clinicopathological study by O'Sullivan et al. (6.2 years),[35] whereas a much longer survival was found for PSP-P patients (11.6 years). Although our PSP-P group consists of only 7 cases, due to the strict use of NINDSSPSP criteria, the difference in survival compared to our RS cases was striking as well. The effect of higher onset-age on survival in the present study was also found in retrospective studies, [20, $21,35]$ whereas our observed predictive value of gender contrasted to a weak or absent effect on survival in several other studies,[18, 20,21] but not all.[35] The prognostic significance of older onset-age in PSP resembles observations made in Alzheimer's disease[36] and Parkinson's disease (PD), whereas there is conflicting evidence regarding effect on prognosis of male gender in PD. [37] A good explanation for lower survival in men with PSP in our study is lacking. The finding might perhaps be explained by differences for gender in co-morbidity at higher age. However, such data are not available in our study. The predictive value of the PSPRS score for survival in PSP patients confirms the first observations made in a tertiary referred cohort of Golbe et al.[21] and also proves its predictive value in a population-based cohort. In line with Golbe's observations, a sharp rise in mortality risk was seen in patients with a PSPRS score above 60. Only the subsections supranuclear ocular motor exam, bulbar exam and gait exam were of prognostic value in our study. The replication of Golbe's findings on the PSPRS has implications for its potential use in clinical trials. 
Shorter survival in FTD patients with a positive family history in this study contrasts with other studies on the natural history of FTD, $[12,13,24,38,39]$ and may suggest a more malignant disease process for hereditary forms. This is especially true for patients with GRN mutations and hereditary FTLD with an unknown genetic defect, both groups exhibiting ubiquitin pathology,[40] whereas MAPT mutations showed a trend towards longer disease duration. $\underline{\text { However, as several mutation carriers were related, we cannot exclude other familial genetic }}$ factors influencing the disease duration within the families. The absence of an association between positive family history and survival in other studies may be explained by a low number of patients or an unknown family history.

The longer survival of F'TLD-tau group than compared to pathologically proven PSP cases supports the hypothesis of a different disease process. The mean disease duration in the present series of 11.1 years is similar to that in the study by Hodges et al. (9.0 years).[24] The shorter survival of tau-positive cases ( 6 years) in the study by Xie et al.[16] can be explained by the inclusion of PSP and CBD cases. Our findings are very similar to the observations made by Hu et al.,[14] which showed that three-repeat FTLD-tau have longer survival than four-repeat FTLDtau and four-repeat controls, comprising PSP and CBD patients, and supports the idea that FTLD-tau patients tend to have a more indolent disease course than PSP.

The observed negative correlation between the severity of glial tau pathology and disease duration in PSP patients is in line with the study by Josephs et al.[22] The severity of oligodendroglial tau pathology in the substantia nigra and caudate and dentate nucleus represented the overall tau pathology reliably in the study by Williams et al, which again correlated negatively with disease duration, [23] and was shown higher in RS than in PSP-P. Due to the absence of PSP-P in our pathological cohort, we could not replicate the latter finding. The correlation between the type and severity of tau pathology indicates that pathophysiological mechanisms determine the disease progression. Small sample size, a semiquantitative method of scoring, and different $M A P T$ mutations with different functional effects may have hampered our 
analysis in F'TLD. The association in F'TLD between shorter survival and abundant tau pathology in basal ganglia in the study by Xie et al.[16] could not be confirmed by our study, and should probably be explained by the inclusion of PSP and CBD cases in their analysis. The best strategy would be to extend the survival analysis to a much larger series of pathologically proven FTD cases, which have been prospectively ascertained during life in order to collect reliable clinical information.

One of the drawbacks of the present study is a selection bias towards typical cases, and therefore missing cases with PSP-P, a subgroup that usually has a longer disease duration and an atypical presentation. Furthermore, as the population of the study consists of cases alive at the time of entry, there may be some degree of survival bias. A final drawback is that there was pathological confirmation in only 24 of 133 of the deceased patients. However the NINDS-SPSP criteria show a good positive predictive value for probable PSP (100\%) and possible PSP (83\%) in patients presenting with parkinsonism, [34] but also in patients presenting with dementia $(96 \%$ for combined possible and probable PSP).[41] Also, no large differences were found between our clinical and our pathological cohort.

In conclusion, this large prospective study showed that survival in PSP is shorter than in FTD. This difference in prognosis was even more pronounced when comparing pathological PSP cases with F'TLD-tau. Within the PSP group, male gender, older onset-age, and higher PSPRS score were independent predictors for shorter disease duration, whereas a positive family history and an older onset-age were associated with a poor prognosis in FTD. The significant effect of diagnosis on survival may suggest that the underlying pathophysiology in PSP is more aggressive than in FTD. This perspective should help clinicians anticipate disease progression of patients with PSP and FTD.

\section{Disclosure}

The authors report no conflict of interest 


\section{Funding}

This study was financially supported by Prinses Beatrix Fonds (grant number 01-0128), the Hersenstichting (project number 13F05(2).14) and stichting Dioraphte (grant number 0701 05).

\section{Copyright license statement}

The Corresponding Author has the right to grant on behalf of all authors and does grant on behalf of all authors, an exclusive licence on a worldwide basis to the BMJ Publishing Group Ltd, and its Licensees to permit this article (if accepted) to be published in Journal of Neurology, Neurosurgery \& Psychiatry and any other BMJPGL products and to exploit all subsidiary rights, as set out in our licence. 


\section{References}

[1] Steele JC, Richardson JC, Olszewski J. Progressive supranuclear palsy. A heterogeneous degeneration involving the brain stem, basal ganglia and cerebellum with vertical gaze and pseudobulbar palsy, nuchal dystonia and dementia. Archives of Neurology. 1964;10:333-59.

[2] Litvan I, Agid Y, Calne D, et al. Clinical research criteria for the diagnosis of progressive supranuclear palsy (Steele-Richardson-Olszewski syndrome): report of the NINDS-SPSP international workshop. Neurology. 1996 Jul;47(1):1-9.

[3] Boeve BF. Links between frontotemporal lobar degeneration, corticobasal degeneration, progressive supranuclear palsy, and amyotrophic lateral sclerosis. Alzheimer Dis Assoc Disord. 2007 Oct-Dec;21(4):S31-8.

[4] Kertesz A, Munoz D. Relationship between frontotemporal dementia and corticobasal degeneration/progressive supranuclear palsy. Dement Geriatr Cogn Disord. 2004;17(4):282-6.

[5] Mackenzie IR, Neumann M, Bigio EH, et al. Nomenclature for neuropathologic subtypes of frontotemporal lobar degeneration: consensus recommendations. Acta Neuropathol. 2009 Jan;117(1):15-8.

[6] Donker Kaat L, Boon AJ, Kamphorst W, et al. Frontal presentation in progressive supranuclear palsy. Neurology. 2007 Aug 21;69(8):723-9.

[7] Wszolek ZK, Tsuboi Y, Uitti RJ, et al. Progressive supranuclear palsy as a disease phenotype caused by the S305S tau gene mutation. Brain. 2001 Aug;124(Pt 8):1666-70.

[8] Morris HR, Osaki Y, Holton J, et al. Tau exon $10+16$ mutation F'TDP-17 presenting clinically as sporadic young onset PSP. Neurology. 2003 Jul 8;61(1):102-4.

[9] de Silva R, Lashley T, Strand C, et al. An immunohistochemical study of cases of sporadic and inherited frontotemporal lobar degeneration using 3R- and 4R-specific tau monoclonal antibodies. Acta Neuropathol. 2006 Apr;111(4):329-40. 
[10] van Swieten J, Spillantini MG. Hereditary frontotemporal dementia caused by Tau gene mutations. Brain Pathol. 2007 Jan;17(1):63-73.

[11] Baker M, Litvan I, Houlden H, et al. Association of an extended haplotype in the tau gene with progressive supranuclear palsy. Hum Mol Genet. 1999 Apr;8(4):711-5.

[12] Borroni B, Grassi M, Agosti C, et al. Survival in frontotemporal lobar degeneration and related disorders: latent class predictors and brain functional correlates. Rejuvenation Res. 2009 Feb;12(1):33-44.

[13] Roberson ED, Hesse JH, Rose KD, et al. Frontotemporal dementia progresses to death faster than Alzheimer disease. Neurology. 2005 Sep 13;65(5):719-25.

[14] Hu W'T, Parisi JE, Knopman DS, et al. Clinical features and survival of 3R and 4R tauopathies presenting as behavioral variant frontotemporal dementia. Alzheimer Dis Assoc Disord. 2007 Oct-Dec;21(4):S39-43.

[15] Kertesz A, McMonagle P, Blair M, et al. The evolution and pathology of frontotemporal dementia. Brain. 2005 Sep;128(Pt 9):1996-2005.

[16] Xie SX, Forman MS, Farmer J, et al. Factors associated with survival probability in autopsy-proven frontotemporal lobar degeneration. J Neurol Neurosurg Psychiatry. 2008 Feb;79(2):126-9.

[17] Testa D, Monza D, Ferrarini M, et al. Comparison of natural histories of progressive supranuclear palsy and multiple system atrophy. Neurol Sci. 2001 Jun;22(3):247-51.

[18] Papapetropoulos S, Gonzalez J, Mash DC. Natural history of progressive supranuclear palsy: a clinicopathologic study from a population of brain donors. Eur Neurol. 2005;54(1):1-9.

[19] Litvan I, Mangone CA, McKee A, et al. Natural history of progressive supranuclear palsy (Steele-Richardson-Olszewski syndrome) and clinical predictors of survival: a clinicopathological study. J Neurol Neurosurg Psychiatry. 1996 Jun;60(6):615-20.

[20] Nath U, Ben-Shlomo Y, Thomson RG, et al. Clinical features and natural history of progressive supranuclear palsy: A clinical cohort study. Neurology. 2003;60(6):910-6. 
[21] Golbe LI, Ohman-Strickland PA. A clinical rating scale for progressive supranuclear palsy. Brain. 2007 Jun;130(Pt 6):1552-65.

[22] Josephs KA, Mandrekar JN, Dickson DW. The relationship between histopathological features of progressive supranuclear palsy and disease duration. Parkinsonism Relat Disord. 2006 Mar;12(2):109-12.

[23] Williams DR, Holton JL, Strand C, et al. Pathological tau burden and distribution distinguishes progressive supranuclear palsy-parkinsonism from Richardson's syndrome. Brain. 2007 Jun;130(Pt 6):1566-76.

[24] Hodges JR, Davies R, Xuereb J, et al. Survival in frontotemporal dementia. Neurology. 2003 Aug 12;61(3):349-54.

[25] Rosso SM, Donker Kaat L, Baks T, et al. Frontotemporal dementia in The Netherlands: patient characteristics and prevalence estimates from a population-based study. Brain. 2003 Sep;126(Pt 9):2016-22.

[26] Clinical and neuropathological criteria for frontotemporal dementia. The Lund and Manchester Groups. J Neurol Neurosurg Psychiatry. 1994 Apr;57(4):416-8.

[27] Williams DR, de Silva R, Paviour DC, et al. Characteristics of two distinct clinical phenotypes in pathologically proven progressive supranuclear palsy: Richardson's syndrome and PSP-parkinsonism. Brain. 2005 Jun;128(Pt 6):1247-58.

[28] Baker M, Mackenzie IR, Pickering-Brown SM, et al. Mutations in progranulin cause taunegative frontotemporal dementia linked to chromosome 17. Nature. 2006 Aug 24;442(7105):916-9.

[29] Rizzu P, Van Swieten JC, Joosse M, et al. High prevalence of mutations in the microtubule-associated protein tau in a population study of frontotemporal dementia in the Netherlands. Am J Hum Genet. 1999 Feb;64(2):414-21.

[30] Skibinski G, Parkinson NJ, Brown JM, et al. Mutations in the endosomal ESCRTIIIcomplex subunit CHMP2B in frontotemporal dementia. Nat Genet. 2005 Aug;37(8):806-8. 
[31] Bronner IF, Rizzu P, Seelaar H, et al. Progranulin mutations in Dutch familial frontotemporal lobar degeneration. Eur J Hum Genet. 2007 Mar;15(3):369-74.

[32] Mata IF, Kachergus JM, Taylor JP, et al. Lrrk2 pathogenic substitutions in Parkinson's disease. Neurogenetics. 2005 Dec;6(4):171-7.

[33] Cairns NJ, Bigio EH, Mackenzie IR, et al. Neuropathologic diagnostic and nosologic criteria for frontotemporal lobar degeneration: consensus of the Consortium for Frontotemporal Lobar Degeneration. Acta Neuropathol. 2007 Jul;114(1):5-22.

[34] Litvan I, Hauw JJ, Bartko JJ, et al. Validity and reliability of the preliminary NINDS neuropathologic criteria for progressive supranuclear palsy and related disorders. J Neuropathol Exp Neurol. 1996 Jan;55(1):97-105.

[35] O'Sullivan SS, Massey LA, Williams DR, et al. Clinical outcomes of progressive supranuclear palsy and multiple system atrophy. Brain. 2008 May;131(Pt 5):1362-72.

[36] Larson EB, Shadlen MF, Wang L, et al. Survival after initial diagnosis of Alzheimer disease. Ann Intern Med. 2004 Apr 6;140(7):501-9.

[37] Post B, Merkus MP, de Haan RJ, et al. Prognostic factors for the progression of Parkinson's disease: a systematic review. Mov Disord. 2007 Oct 15;22(13):1839-51; quiz 988.

[38] Grasbeck A, Englund E, Horstmann V, et al. Predictors of mortality in frontotemporal dementia: a retrospective study of the prognostic influence of pre-diagnostic features. Int J Geriatr Psychiatry. 2003 Jul;18(7):594-601.

[39] Pasquier F, Richard F, Lebert F. Natural history of frontotemporal dementia: comparison with Alzheimer's disease. Dement Geriatr Cogn Disord. 2004;17(4):253-7.

[40] Seelaar H, Kamphorst W, Rosso SM, et al. Distinct genetic forms of frontotemporal dementia. Neurology. 2008 Oct 14;71(16):1220-6.

[41] Lopez OL, Litvan I, Catt KE, et al. Accuracy of four clinical diagnostic criteria for the diagnosis of neurodegenerative dementias. Neurology. 1999 Oct 12;53(6):1292-9. 


\section{Figure legends}

Figure 1 Kaplan Meier survival curve for PSP and FTD patients

Figure 2 Disease duration of pathologically proven PSP cases according to PSP-tau score (Spearman's rho $-0.44, \mathrm{p}=0.045$ ) 


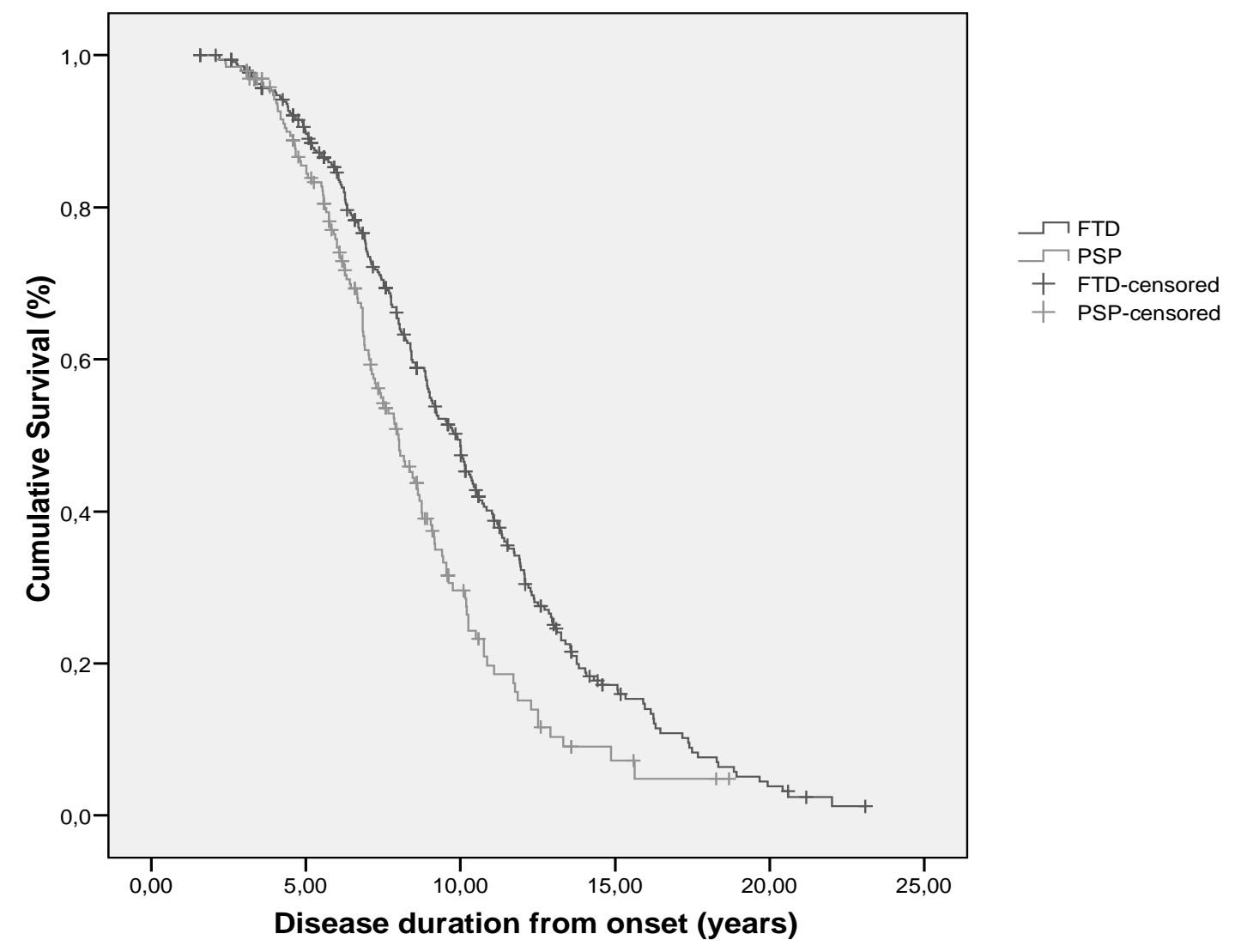




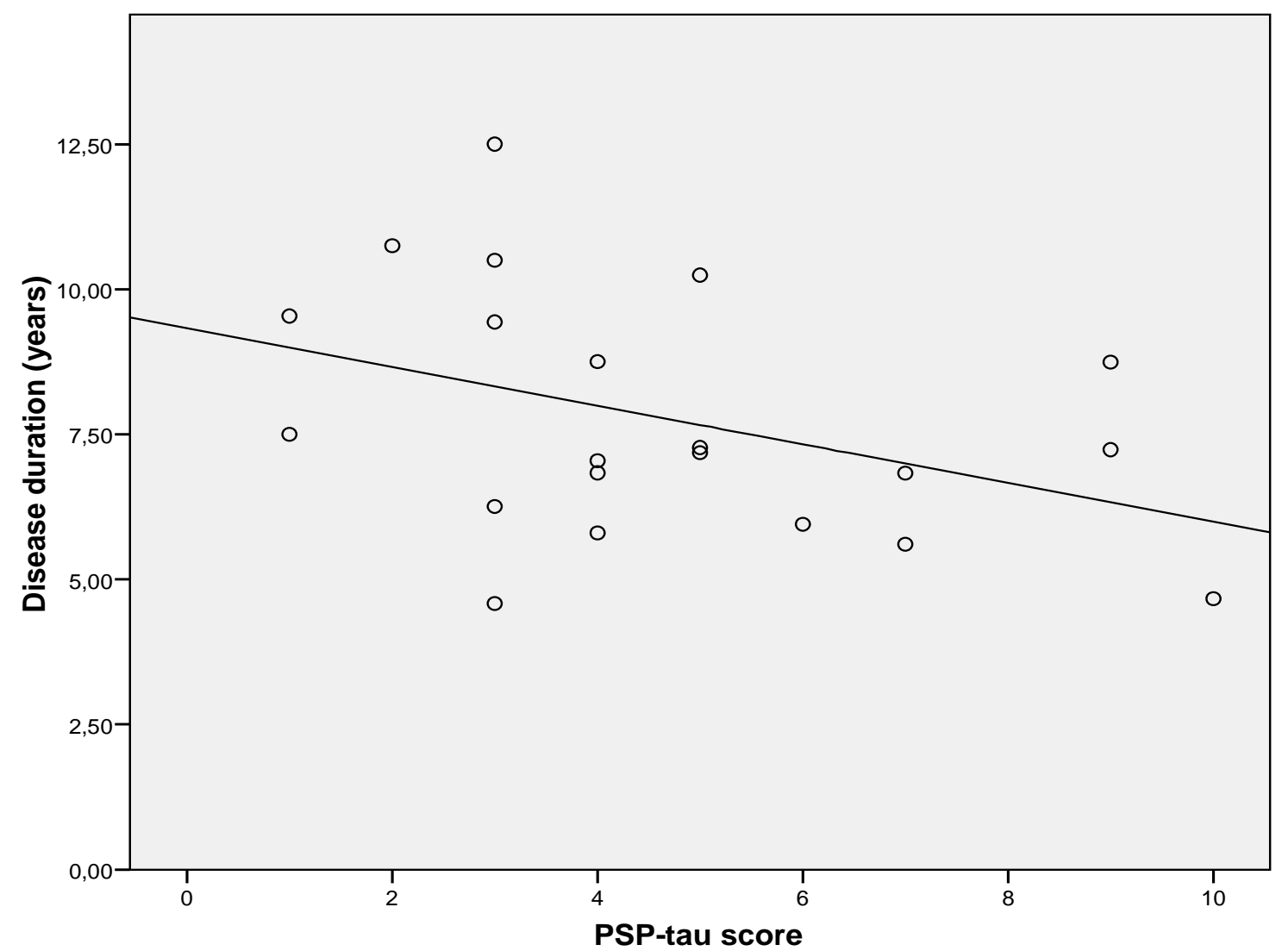

